\title{
Pharma partners with efforts to pool patient genotype and phenotype data
}

A partnership between Pfizer and 23andMe, announced in August, sheds light on the value biopharma companies are beginning to attach to combining genomics with a range of patient-derived phenotypic data. The interest is a boost for the direct-to-consumer business models of for-profit firms like 23andMe and PatientsLikeMe. Such moves are also in lockstep with government-driven initiatives. These include the public, multinational consortium 1000 Genomes Project (Nat. Biotechnol. 26, 256, 2008) and the UK's nascent 100,000 Genomes Project, which recently received a boost in funding through its manager, Genomics England.

The arrangement with 23 andMe, of Mountain View, California, calls for it to recruit 10,000 new individuals with inflammatory bowel disease (IBD) for an observational study (one in which no treatment is given), in part through outreach to disease foundations and Pfizer's existing network of collaborators at hospitals. The participants will receive the company's genome sequencing service and provide survey information including their experiences having IBD, a set of immune disorders that includes Crohn's disease and ulcerative colitis, and treatments they have received. 23andMe will analyze the data and provide it to Pfizer. Enrollees will sign consent forms specifically designed to enable their individual level data to be shared with the New York-based big pharma.

The heterogeneity, complexity and episodic nature of IBD, which is characterized by intermittent flares and periods of pain and diarrhea, makes the ability to survey patients particularly important: most of the approvable clinical endpoints are based on questionnaires like the Crohn's Disease Activity Index. "We know that the genetics of IBD is quite rich," comprising a constellation of 50-60 pathways directly associated with it, says Pfizer's head of biotherapeutics R\&D, Jose Carlos Gutierrez-Ramos. "It's a question of putting the pieces of the puzzle together but the pieces are there," he says.

The partnership's primary goal is to use the data to try to get a better handle on the degree to which various subgroups of patients respond to Pfizer's pipeline of IBD drugs, which include an inhibitor of Janus-associated kinase 3, Xeljanz (tofacitinib) and four biologics-all five are in late-stage trials for treatment of Crohn's and/or ulcerative colitis and target different pathways. Through its online service, 23andMe also will give Pfizer greater engagement with patients. "We can recontact a subgroup with a phenotype and ask them for participation in a study," Gutierrez-Ramos says. The data will also help confirm patients' responsiveness to the various pathway-directed therapies. "We see this collaboration transforming all the way from discovery through clinical development," he says. "It is a very important collaboration for us."

23andMe has previously conducted similar but smaller projects: with S. San Francisco, California's Genentech to study patients who had taken the drug Avastin (bevacizumab) for metastatic cancer and with Janssen, a Johnson \& Johnson company in Raritan, New Jersey, in rheumatoid arthritis. Cambridge, Massachusetts-based PatientsLikeMe, which collects real-world data from individuals about their experiences with disease and analyzes them for use by researchers and companies, also inked a five-year, oncology-focused agreement with Genentech in April that gives Genentech access to its patient network and capabilities.

The sharpened focus downstream on patient data is a logical consequence of technology evolution. Genomics is reaching the point where sequencing is not that expensive, says Lisa Brooks, who manages the Genetic Variation Program for the Division of Genome Sciences at the National Human Genome Research Institute (NHGRI) and oversees the 1000 Genomes Project. "But interacting with patients and getting clinical phenotype information is quite expensive," she says. "23andMe has a model where they do that through the patients themselves."

23andMe may have been warned by the US Food \& Drug Administration in November 2013 that the marketing of its service was in violation of regulations (Nat. Biotechnol. 32, 1, 2014), but NHGRI had no trouble authorizing a \$1.4-million, phase 2, Small Business Innovation Research grant for the firm in August. The grant is helping 23andMe develop survey tools, gather longitudinal and cognitive data, analyze data and test methods for providing external researchers and companies with access to the database. "This was a pretty straightforward award," says Brooks.

From its experiences with industry partners and as part of consortia for disease studies in areas including Parkinson's disease, sarcoma and myeloproliferative neoplasms, 23andMe has refined its ability to connect with patients, who participate online from home. "They are bringing to disease studies a lot of samples and a lot of data," Brooks says. "That's what's really needed."

Although distinct from the 23andMe directto-consumer project, the 100,000 Genomes Project is similarly a combination of genomic and patient-centric data, linking individual medical records and in the process, gathering

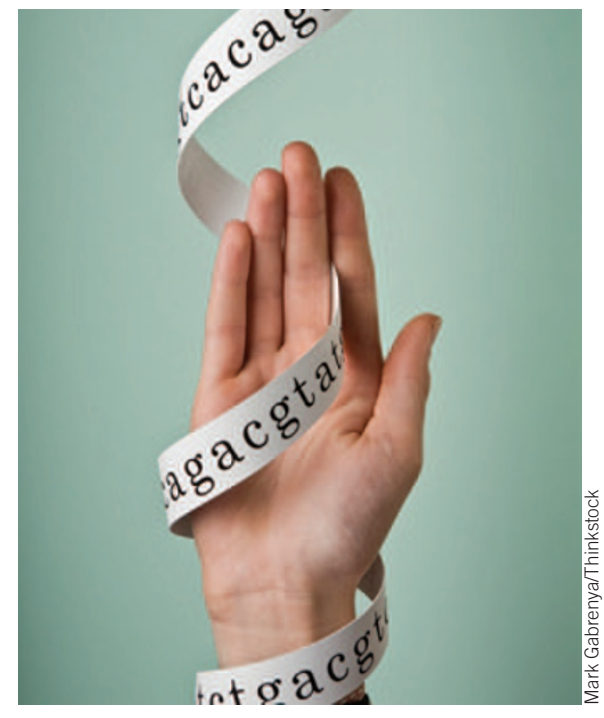

The race is on to gather as much genotype and phenotype data as possible from patients with conditions like rheumatoid arthritis or IBD.

information on a variety of illnesses.

From its base in Queen Mary University of London, Genomics England is doling out over $£ 300$ million (more than $\$ 500$ million) in new investments for the project, which will sequence individuals with cancer, rare diseases and infectious diseases. More than half the money will come from Illumina of San Diego, which will supply sequencing equipment in a new facility at The Sanger Center in Cambridge and will recoup almost half its investment through service fees. Rounding out the contributions are The Wellcome Trust, which is funding construction of the new sequencing center (£27 million/\$43.7 million), the Medical Research Council for data storage and interpretation ( $£ 24$ million/\$39.6 million), and the National Health Service (NHS) for patient and sample recruitment (up to $£ 20$ million/ $\$ 32.6$ million). Built into the program will be receipt of information about treatment and outcomes. NHS will link, with the patients' permission, to their medical records in primary care in hospitals, family physicians' office in hospitals and also to a registry Genomics England will hold, to provide a comprehensive picture of patients and their outcomes over their life course. "We will also be receiving information about treatment and outcomes from the registry and continually be updating data from the electronic format," says chief scientist Mark Caulfield. The group will also have the ability, with their consent, to approach patients four times a year for research opportunities.

Mark Ratner Boca Raton, Florida 